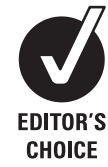

CHOICE

\title{
Development of an integrated pancreatic disease service
}

\author{
James A Jupp, ${ }^{1}$ Colin D Johnson, ${ }^{2}$ Fanny W Shek, ${ }^{3}$ David R Fine ${ }^{3}$
}

\begin{abstract}
'University of Southampton, School of Medicine, Southampton, UK ${ }^{2}$ University of Southampton and Southampton University Hospitals NHS Trust, Southampton, UK ${ }^{3}$ Department of Gastroenterology, Southampton University Hospitals NHS Trust, Southampton, UK
\end{abstract}

\section{Correspondence to}

Dr D R Fine, Department of Gastroenterology, Southampton University Hospitals NHS Trust, Southampton General Hospital, Mailpoint 255, Tremona Road, Southampton S016 6YD, UK; david.fine@suht.swest.nhs.uk

Accepted 10 December 2010 Published Online First 9 January 2011

\begin{abstract}
An integrated pancreatic disease unit needs to deliver high-quality care both to patients with malignant and non-malignant pancreatic disease. The regionalisation of pancreatic cancer services which followed the publication of policy frameworks by the Department of Health and NHS executive led to the development of diseasesite-specialised high-volume multidisciplinary teams. As the majority of patients with pancreatic cancer are not suitable for surgery, partner hospitals within a region need to provide access to a wide range of non-surgical treatment. The implementation of such working may require pooling of local resources to create networks of equivalence to tertiary centres. The provision of care to non-malignant pancreatic disease can benefit from this type of working and services can be modelled on, and integrate with, cancer services. One way of achieving this is to establish working groups based upon diseases rather than traditional departments, which can deliver standardised and optimal care with a patient-centred approach. However, this poses a number of potential problems.

This review examines how an integrated pancreatic unit may be developed in district general and larger hospitals, and also describes our experience in developing such a unit.
\end{abstract}

\section{Introduction}

An integrated pancreatic unit should deliver high-quality patient-centred care both for malignant and for non-malignant pancreatic disease. It can involve a wide variety of specialists depending on local resources (table 1). Although pancreatic disease accounts for a small proportion of the workload of a gastrointestinal unit, bringing cases together under the care of a pancreatic team should improve patient experience and outcome. While cancer surgery is limited to cancer centres, the other resources should be available within most district general hospitals (DGHs); pulling them together into a single service, however, requires dedication and funding. This review examines how this can be achieved in DGHs and larger centres.

The creation of cancer networks has had mixed effects on pancreatic patients as a group. Patients with cancer have benefited as described below. Pancreatic patients without cancer may benefit indirectly from the existence of the network, but may not benefit directly from the network's resources. As with all diseases outside a national service framework programme, it is vital that clinicians are advocates for the needs of their non-cancer patients and ensure that cancer targets do not compromise the care of non-cancer patients.

\section{Creation of specialist pancreatic surgical centres}

In 1995, the Expert Advisory Group on cancer published a policy framework for the organisation and management of cancer (the 'Calman-Hine' report), ${ }^{1}$ proposing that services should be organised according to the following principles:

- All patients should have access to a uniformly high quality of care.

- Public and professional education assists early diagnosis.

- Patients, families and carers should be given clear information about treatment options.

- The development of cancer services should be patient-centred with the primary care team providing the central and continuing element of care.

- Monitoring of treatments and outcomes is essential.

It recommended the establishment of cancer networks offering patients specialised care close to home or, for more complex or rarer disease, referral to specialised units. Evidence that higher case volume is associated with better surgical outcome for rarer and high-risk cancers such as pancreatic cancer ${ }^{2-4}$ led to the policy of 
Table 1 Potential components of an integrated pancreatic disease unit

Pancreatic surgeons

Gastroenterologists with a specialist interest in pancreatic disease

Radiologists with a specialist interests in gastrointestinal disease and interventional radiology

Oncologists (medical and radiotherapy)

Histopathologists with a specialist interest in gastrointestinal disease

Endoscopists and radiologists with skills in ERCP and EUS

Nurse specialists, incorporating both cancer and non-cancer roles

Palliative care team*

Pain management team*

Dieticians and nutrition support teams

Diabetologists and diabetes specialist nurses

Multidisciplinary team coordinator

Psychological medicine*

Geneticists*

Anaesthetist/intensivist*

Paediatricians*

Physiotherapy and occupational therapy*

Medical social worker*

Audit coordinator

Clinical trials coordinator

An MDM chairperson, who may be from another subspecialty

*These disciplines are not yet fully integrated with the pancreatic disease unit in Southampton, although their services are available.

ERCP, endoscopic retrograde cholangiopancreatography; EUS, endoscopic ultrasound; MDM, multidisciplinary meeting.

limiting cancer surgery to units capable of supporting disease-site-specialised high-volume multidisciplinary teams. Initial work concentrated on the development of specialist oncology, rather than specialist surgery. Then in 2001 guidance for the management of upper gastrointestinal tumours (NHS Executive, guidance on commissioning cancer services) ${ }^{5}$ provided a framework for the organisation of regional surgical services, which now represent the 'gold standard' for care across the UK, ensuring optimal treatment is delivered based upon best clinical practice.

\section{Regional multidisciplinary working}

A regional service centres on review of patients at a multidisciplinary meeting (MDM), usually weekly, at the central site at which surgery is performed. This should link seamlessly with subregional MDMs either by attendance of key individuals from the regional centre-for example, pancreatic surgeons, specialist pancreatic oncologists and radiologists, at subregional MDMs; attendance of clinicians within the region at the central MDM; or by videoconferencing between peripheral hospitals and the tertiary centre. All patients with a suspected pancreatic malignancy should be discussed with key members of the central MDM regardless of review elsewhere beforehand.

The linchpin of the regionalised multidisciplinary process is the coordinator who must ensure there is close liaison with subregional MDMs. In addition, their role in collating essential information such as imaging, histology and disease registry data is central to the smooth and efficient running of the process and thus delivery of high-quality care. They must also have close working relationships with the clinical nurse specialists and lead clinician, and ensure decisions made at meetings are disseminated to all parties involved in delivering care, in particular to primary care doctors and booking clerks.

The benefits claimed for regionalised multidisciplinary working are numerous: first, they improve patient outcomes. This is related to case review by individuals with specialist knowledge so that the correct diagnosis, determination of resectablility and timing/ sequence of treatment are optimal. Pawlik and colleagues recently showed that $23.6 \%$ of patients had a change in their recommended management following clinical review of cases by the central multidisciplinary tumour board. ${ }^{6}$ Second, throughput of an appropriate number of patients allows skills to be maintained, thus ensuring the best quality of care is available. Third, regional collaborative working allows for standardisation of care-for instance, of cross sectional imaging and chemotherapy protocols. Finally, it disseminates knowledge about national and local support groups and facilitates clinical trial recruitment. Recent evidence suggests that mortality from pancreatic cancer may be falling, perhaps as a result of improvements in treatment. ${ }^{7}$ These improvements include a reduction in surgical mortality rates, more thorough investigation and higher resection rates, ${ }^{89}$ the use of postoperative adjuvant chemotherapy ${ }^{10}$ and more effective palliative chemotherapy. ${ }^{11}$

Clinical nurse specialists (CNS) are key elements of the service who manage the patient journey, support carers and understand the special needs of patients with pancreatic cancer (such as pancreatic enzyme replacement, pain control, dietary requirements, impact of surgery and chemotherapy). The multidisciplinary specialists at the regional centre provide training for CNS working throughout the region, and the highly specialised CNS at the regional centre play an invaluable educational role during regular contact with local CNS. The role of the cancer nurse specialist is therefore of great importance in improving outcomes for patients and has been economically justified. ${ }^{12}$

\section{Integration of regional pancreatic services}

Many patients with pancreatic cancer present with advanced disease and only $10-15 \%$ are suitable for surgery. Given the low resectability rate it is vital that all hospitals provide access to non-surgical treatment and specialised coordinated care. When certain 
non-surgical elements are not available in hospitals within a region, pooling of resources is necessary. The creation of satellite centres and cross-site working can allow provision of all aspects of care, in all areas. In particular, the early involvement of palliative care and paramedical services assists in making the patient journey as smooth as possible. The development of multidisciplinary teams, and regional referral centres has largely achieved these aims for pancreatic cancer.

\section{Non-malignant pancreatic disease and neuroendocrine tumours}

The working patterns of endoscopists, surgeons and oncologists dealing with cancer are usually in separate spheres of activity, which link with each other at the MDM, in conjunction with radiology and pathology. Integration at local and regional level is mainly through discussions at the MDM. For other pancreatic diseases, however, there is benefit in combined clinical care, particularly between the surgeon and gastroenterologist. The elements vary with the disease, but team working and covering all the patients' needs are common themes. Although chronic pancreatitis is a condition largely treated on an outpatient basis, with few inpatients seen each year (approximately 30 per million hospital discharges per year in a DGH),${ }^{13}$ acute pancreatitis presents a higher inpatient workload. The members of the pancreatic team therefore have differing levels of commitment to each.

Acute pancreatitis is usually managed by surgeons in the UK. National guidelines recommend that large hospitals establish a single surgical team, preferably on one ward, to manage all cases. Smaller hospitals should have agreed management guidelines and a lead pancreatitis surgeon to advise on difficult cases. Key elements are prediction of severity and identification of gallstones: patients predicted to have severe disease by APACHE-II or other scoring systems can be identified and transferred to a high-care area early in their admission; patients with gallstones should have early endoscopic retrograde cholangiopancreatography (ERCP) if merited or definitive treatment booked before discharge. ${ }^{14}$ The establishment of a multidisciplinary ward round (table 2) is also beneficial as nonsurgical problems may be dealt with and followed-up as appropriate.

Patients with chronic pancreatitis and recurrent acute pancreatitis are usually scattered through medical and surgical clinics. We recommend establishing a single multidisciplinary clinic for these patients, the frequency of the clinics depending on the workload. The aims are to establish diagnosis; treat rare causes; optimise nutritional and metabolic status; optimise analgesia; give psychological support; treat addiction and offer surgery where appropriate (table 3). Ideally, all elements should be available in a 'one-stop' visit, but in practice this is hard to achieve.
Table 2 Multidisciplinary pancreatic ward round: components, roles and partners*

\begin{tabular}{|c|c|c|}
\hline Member & Role & Partners* \\
\hline Surgeon & General management + surgery & Existing resource \\
\hline Gastroenterologist & $\begin{array}{l}\text { 'Medical' problems } \\
\text { Outpatient non-surgical problems }\end{array}$ & Existing resource \\
\hline Pancreatic CNS* & $\begin{array}{l}\text { Patient support } \\
\text { Communication } \\
\text { Organisation }\end{array}$ & Cancer service \\
\hline Diabetologist/CNS & $\begin{array}{l}\text { Metabolic problems } \\
\text { Insulin control } \\
\text { Outpatient follow-up }\end{array}$ & Diabetes service \\
\hline Intensivist & Management of critically ill patients & Existing resource \\
\hline Addiction CNS & $\begin{array}{l}\text { Assess addictions } \\
\text { (alcohol and other) }\end{array}$ & $\begin{array}{l}\text { Hepatology } \\
\text { Community } \\
\text { addiction } \\
\text { programmes }\end{array}$ \\
\hline
\end{tabular}

*Partners are services which might join with a pancreatic unit in a business case.

CNS, clinical nurse specialist.

Table 3 Multidisciplinary pancreatic clinics: components, roles and partners*

\begin{tabular}{|c|c|c|}
\hline Member & Role & Partner* \\
\hline Gastroenterologist & $\begin{array}{l}\text { Overall investigation } \\
\text { and management }\end{array}$ & Existing resource \\
\hline Dietician & $\begin{array}{l}\text { Nutrition + enzyme } \\
\text { treatment }\end{array}$ & Nutrition. Cystic fibrosis. \\
\hline Pain specialist/CNS & Pain management & $\begin{array}{l}\text { Other services with } \\
\text { chronic pain patients, for } \\
\text { example, IBS, gynaecology }\end{array}$ \\
\hline Psychological medicine & $\begin{array}{l}\text { Psychological support, } \\
\text { pain management, } \\
\text { addiction }\end{array}$ & $\begin{array}{l}\text { Other services with } \\
\text { chronic pain patients, for } \\
\text { example, IBS, gynaecology }\end{array}$ \\
\hline Addiction specialist & $\begin{array}{l}\text { Managing addictive } \\
\text { behaviour }\end{array}$ & $\begin{array}{l}\text { Community addiction } \\
\text { programmes }\end{array}$ \\
\hline Surgeon & Surgical management & Existing resource \\
\hline
\end{tabular}

*Partners are services which might join with a pancreatic unit in a business case.

CNS, clinical nurse specialist; IBS, irritable bowel syndrome.

Although pancreatic neuroendocrine tumours are malignant, their rarity (table 4) poses similar problems in the development of a multidisciplinary service. Their complexity deserves specialised coordinated management by a dedicated team linked to pancreatic carcinoma services. Cases should therefore preferably be pooled in a regionalised neuroendocrine clinic. The chronic relapsing nature of the disease can produce varied endocrine and metabolic problems, and cause widespread metastases. The team will consequently require additional support from an endocrinologist, specialist oncologist and nuclear medicine doctor, particularly as newer biological, chemo-radiological and radionucleotide treatments become available.

Cystic fibrosis (CF) is generally managed in regional units. Gastroenterologists and dieticians are 
Table 4 Incidence of pancreatic diseases related to typical hospital catchment area populations

\begin{tabular}{lllcc}
\hline Condition & Reference & No/100K & No/250K & No/500K \\
\hline Acute pancreatitis & Ellis et al15 & 56.5 & 141 & 283 \\
Acute pancreatitis & Roberts et all & 22.4 & 56 & 112 \\
Chronic pancreatitis & Lankish et al7 & 6.4 & 16 & 32 \\
NET pancreas & Oberg and Eriksson & 3 & 5 \\
\hline
\end{tabular}

NET, neuroendocrine tumours.

an essential element in this service, but pancreatic surgery is rarely indicated. Advice should always be sought from the regional unit if a patient with $\mathrm{CF}$ has pancreatitis or a significant gastrointestinal problem.

\section{Strategies for creating an integrated pancreatic disease unit}

Changes in working practice may be relatively easy to implement; objections may be raised but these can usually be countered by thought and preparation. The obstacles to creating a pancreatic unit are lack of perception of need; resistance to subspecialisation and lack of resources. These can be overcome but a lot of effort is needed. Perception of need relates to the relative rarity of pancreatic disease (table 4). While individual patients may be high consumers of care, if they are widely distributed their impact on individual clinicians is limited. Similarly, the fact that they are receiving suboptimal care may not be appreciated. Pointing this out to both clinicians and managers may be all that is required, but often an audit of admissions and resources consumed is good evidence, and essential for compiling a business case.

Resistance to subspecialisation is understandable. Clinicians fear becoming de-skilled and losing prestige. Most surgeons are already subspecialised, but many gastroenterologists are generalists. The arguments for subspecialisation apply to all other diseases, therefore a clinician arguing for specialist pancreatology must allow colleagues to establish similar specialist interests. Establishment of a new MDM also requires good negotiating skills as pathologists and radiologists are particularly hardpressed by cancer MDMs and find it hard to fit new meetings into their job plans. Consequently, it may prove more feasible to extend the cancer MDM to allow review of non-malignant cases as the numbers will be small.

Resources required to facilitate such a change may be negligible as reorganisation of existing wards or clinics is usually cost-neutral and may improve efficiency. Procurement of additional resources, however, will require a business case. The input required is usually a fraction of a working time equivalent/session for each specialist so making a wider case across your specialty or division may help. Possible co-applicants or 'partners' are listed in tables 2 and 3. Managers should help in this process, which may involve local commissioners, to facilitate cross-site working. Extending services by encouraging the foundation of support groups is also good practice and may bring the benefit of charitable funds.

\section{The Southampton experience}

Southampton General Hospital is the regional centre for pancreatic cancer surgery in the Central South Coast Cancer Network. It serves approximately 3.8 million people and has close liaison with 11 regional hospitals. The Southampton MDM is an integrated pancreatic/biliary/hepatology meeting, chaired by a consultant hepatologist. The average case volume is $40 /$ week and the meeting lasts $3-31 / 2 \mathrm{~h}$. We have attempted to split the meeting into pancreatic and non-pancreatic components, but this has not worked because of overlapping interests within the group (eg, some hepatologists provide ERCP; some surgeons undertake both liver and pancreatic surgery). A regionalised service was developed incrementally over several years and was formally established in 2007. It functions in three main areas: the Southampton MDM reviews all cases referred for specialist treatment, and in addition, pancreatic surgeons from Southampton attend two outside MDMs to improve communication and discussion within subregional networks. In one hospital, clinicians or CNS from two other hospitals attend in person; in the other, videoconferencing to three other sites allows discussion of each case with the referring clinicians. Regional clinicians are also encouraged to visit the Southampton MDM and a number do so regularly. Our (unpublished) audit of these arrangements has shown that videoconferencing or face-to-face contact are associated with higher referral rates than those seen from MDMs acting independently and referring cases according to agreed protocol. This network of MDMs required Department of Health funding for purchase of videoconference equipment and the employment of administrative and specialist nursing staff in most partner hospitals. It has also required agreement of specialists within the region to adopt new policies; this has been instrumental in crystallising the primary aim of delivering highquality care. 
Our multidisciplinary approach to the care of nonmalignant pancreatic disease preceded the centralisation of pancreatic cancer surgery. In the mid-1990s we set up a rapid access clinic for jaundiced patients with the aim of streamlining assessment and initial management. The clinic consists of two slots held open in two general surgery clinics with matching slots for ultrasound examination. Patients are then seen with their ultrasound result: those with dilated ducts are scheduled for admission for therapeutic ERCP, with a preceding CT scan if necessary; and those with normal ducts are seen immediately in a simultaneously held hepatology clinic. Its confirmation of effectiveness by internal audit led to this pattern of assessment being incorporated into the cancer waiting time initiative ( 2 week wait clinic).

Following the success of the jaundice clinic, in 1999 two of us (CDJ and DRF) established a joint medical/ surgical clinic for chronic and recurrent acute pancreatitis. This was unfunded, but led to better patient care and improved decision-making, particularly in surgery for benign disease. In 2007 and 2008, 41 out of 200 elective pancreatic operations performed were for benign disease-mainly chronic pancreatitis and pseudocysts. In 2002 we extended joint working to inpatient management with a weekly joint round of all pancreatic patients. This particularly aided routeing of non-surgical patients to the joint clinic and liaison over ERCP. With the goodwill of our colleagues in radiology and surgery we were also able to establish a pancreaticobiliary MDM, again unfunded. Therefore, when centralisation was proposed we already had a multidisciplinary team which was further enhanced by the funding of specialist nurses, a dietician and MDM support. More recently, we have added a diabetologist to our inpatient and outpatient joint service. We now offer non-cancer inpatient and outpatient services to our own catchment area and to patients referred from within the region for complex problems. The outpatient service, especially, is staffed more by gastroenterologists than surgeons, in contrast to services for malignant disease, but runs according to the same principles.

The establishment of working groups based upon diseases rather than traditional departments also led to the development of a joint medical/surgical neuroendocrine clinic and the funding of a gastroenterologist at the CF clinic. The specialist nurses have established a 4-monthly meeting of the Wessex Pancreas and Bile Duct Cancer Support Network to enable patients and carers to meet others in a similar situation and share experiences.

An ethos of research and progressive development of services is now flourishing with each year seeing improvements in integrated care. We hold a 3 -monthly review and audit meetings to examine our performance as measured by clinical outcomes, meeting targets and research performance (funding
Table 5 Numbers of surgeons and procedures performed in Wessex Region in 2003 before centralisation and in 2007 after centralisation

\begin{tabular}{lcc}
\hline & $\mathbf{2 0 0 3}$ & $\mathbf{2 0 0 7}$ \\
\hline Surgeons & 7 & 3 \\
All procedures & 67 & 120 \\
Tumour resections in Southampton & 27 & 100 \\
Tumour resections elsewhere in region & 27 & 0 \\
Intensive care admissions (\% of all cases) & 100 & 12 \\
Mean critical care stay (days) & 1.9 & 3.3 \\
Deaths recorded, $\mathrm{n}$ (\%) & $2(4)$ & $3(3)$ \\
\hline
\end{tabular}

achieved and papers published). Cross-specialty working has allowed a seamless transfer of patients between the disciplines ensuring high-quality treatment and appropriate follow-up. We have also noted an increased resection rate of pancreatic tumours in our catchment area after centralisation of surgical services, no increase in postoperative mortality, which remains below 4\%, and reduced use of intensive care, suggesting that more patients have benefited from appropriate treatment (table 5). However, all facets that encompass a complete unit have still to be accomplished and the integration of more specialties is required. In particular, we are still working to bring chronic pain services and psychological medicine into our service and need to improve links with palliative care and genetics (table 1).

\section{Summary}

Development of an integrated pancreatic disease unit encompasses many disciplines and crosses many traditional boundaries within hospitals. The emphasis at present is often on delivery of care to patients with malignant pancreatic disease, whereby changes in working appear to be improving outcomes. This should not be detrimental to patients with non-malignant pancreatic disease, whose symptoms and life expectancy may be equally poor. The focus of all care should therefore centre on the patient and draw in appropriate resources to facilitate delivery of optimal treatment. This may only be possible if hospitals serving smaller catchment areas create networks of equivalence to tertiary centres and gain access to a number of desirable specialist services. The essence of such working involves breaking down boundaries between disciplines and communicating effectively to achieve a common goal of providing the best healthcare, in the most accessible location, to the most people. This can allow the creation of a sustainable, diverse, educationally fulfilling and economically viable system to benefit all patients with pancreatic disease.

Competing interests None.

Provenance and peer review Commissioned; externally peer reviewed. 


\section{References}

1. Department of Health. A Policy Framework for Commissioning Cancer Services. A Report by the Expert Advisory Group on Cancer to the Chief Medical Officers of England and Wales. London: Department of Health, 1995.

2. Birkmeyer JD, Warshaw AL, Finlayson SR, et al. Relationship between hospital volume and late survival after pancreaticoduodenectomy. Surgery 1999;126:178-83.

3. Hillner BE, Smith TJ, Desch CE. Hospital and physician volume or specialization and outcomes in cancer treatment: importance in quality of cancer care. J Clin Oncol 2000;18:2327-40.

4. Andrén-Sandberg A, Neoptolemos JP. Resection for pancreatic cancer in the new millennium. Pancreatology 2002;2:431-9.

5. NHS Executive. Guidance on Commissioning Cancer Services. Improving Outcomes in Upper Gastrointestinal Cancer. London: Department of Health, 2001.

6. Pawlik TM, Laheru D, Hruban RH, et al. Evaluating the impact of a single-day multidisciplinary clinic on the management of pancreatic cancer. Ann Surg Oncol 2008; 15:2081-8.

7. Fitzsimmons D, Osmond C, George S, et al. Trends in stomach and pancreatic cancer incidence and mortality in England and Wales, 1951-2000. Br J Surg 2007;94:1162-71.

8. Neoptolemos JP, Russell RC, Bramhall S, et al. Low mortality following resection for pancreatic and periampullary tumours in 1026 patients: UK survey of specialist pancreatic units. UK Pancreatic Cancer Group. Br J Surg 1997;84:1370-6.

9. Bachmann MO, Alderson D, Peters TJ, et al. Influence of specialization on the management and outcome of patients with pancreatic cancer. Br J Surg 2003;90:171-7.
10. Neoptolemos JP, Stocken DD, Friess H, et al. A randomized trial of chemoradiotherapy and chemotherapy after resection of pancreatic cancer. N Engl J Med 2004;350:1200-10.

11. Burris HA 3rd, Moore MJ, Andersen J, et al. Improvements in survival and clinical benefit with gemcitabine as firstline therapy for patients with advanced pancreas cancer: a randomized trial. J Clin Oncol 1997;15:2403-13.

12. Pollard CA, Garcea G, Pattenden CJ, et al. Justifying the expense of the cancer Clinical Nurse Specialist. Eur J Cancer Care (Engl) 2010;19:72-9.

13. Johnson CD, Hosking S. National statistics for diet, alcohol consumption, and chronic pancreatitis in England and Wales, 1960-88. Gut 1991;32:1401-5.

14. Working Party of the British Society of Gastroenterology; Association of Surgeons of Great Britain and Ireland; Pancreatic Society of Great Britain and Ireland; Association of Upper GI Surgeons of Great Britain and Ireland. UK guidelines for the management of acute pancreatitis. Gut 2005;54(Suppl 3):iii1-9.

15. Ellis MP, French JJ, Charnley RM. Acute pancreatitis and the influence of socioeconomic deprivation. Br J Surg 2009; 96:74-80.

16. Roberts SE, Williams JG, Meddings D, et al. Incidence and case fatality for acute pancreatitis in England: geographical variation, social deprivation, alcohol consumption and aetiology-a record linkage study. Aliment Pharmacol Ther 2008;28:931-41.

17. Lankisch PG, Assmus C, Maisonneuve P, et al. Epidemiology of pancreatic diseases in Lüneburg County. A study in a defined german population. Pancreatology 2002;2:469-77.

18. Oberg K, Eriksson B. Endocrine tumours of the pancreas. Best Pract Res Clin Gastroenterol 2005;19:753-81. 